\title{
Genetic Diversity Among Colletotrichum falcatum Isolates Causing Red Rot of Sugarcane in Subtropical Region of India
}

\author{
Ratinderbir KAUR ${ }^{1 *}$, Bipen KUMAR ${ }^{1}$, Yogesh VIKAL ${ }^{2}$, Gulzar S. SANGHERA ${ }^{1 *}$ \\ 'Punjab Agricultural University, Regional Research Station, Kapurthala, 144601, Punjab, India \\ ${ }^{2}$ School of Agricultural Biotechnology, Punjab Agricultural University, Ludhiana, Punjab, India; \\ ratinder@pau.edu and sangheragulzar@pau.edu ('corresponding authors)
}

\begin{abstract}
Silver Genetic diversity of Colletotrichum falcatum causing red rot of sugarcane was assessed based on morphological, pathological and molecular characteristics especially from sub-tropical Indian conditions. Sixteen isolates of this pathogen were collected based on the extensive survey on prominent varieties grown in the region along with some elite selections. Morphological observations (colony colour, mycelium pattern and sporulation) grouped the isolates into two distinct types (C1: light type and C2: dark type). However, quantitative data on colony diameter showed five clusters for these isolates. Pathogenic characterization of these isolates on fourteen standard differentials formed six groups, ingroup 1: (CF-Pb-1) isolates Cf-157, Cf-249 and Cf-248 were the most virulent while group 6 (CF-Pb-6) isolates Cf-60 and Cf- 247 were the least one. The genetic relatedness among the isolates using Random Amplified Polymorphic DNA (RAPD) analysis revealed sufficient molecular polymorphism, which in turn confirmed the variation in virulence of different isolates. The data categorized different isolates into two major clusters and five independent lineages. Polymorphic information content (PIC) ranged from 0.701 to 0.929 . Isolate Cf- 223 was found to be genetically most diverse among all the isolates. Present study inferred that morphological grouping of most of the isolates showed positive correlation with the pathogenic variability while molecular diversity did not showed such associations.
\end{abstract}

Keywords: Colletotrichum falcatum, sugarcane, variability, diversity, molecular marker

\section{Introduction}

Sugarcane (Saccharum spp.) is one of the most important agro-industrial crops of India next to textile industry. It is is grown in both tropical and sub-tropical belts of the country, later has a major concern with respect to the total pool of sugar production in the country. Of the various biotic stresses of sugarcane, red rot (caused by Colletotrichum falcatum) is a devastating fungal disease posing a serious threat to sugarcane cultivation in India (Freeman, 1997; Alexander and Viswanathan, 1996). This disease causes losses in both cane yield and sugar recovery to the tune of 29.07\% and 30.8\%, respectively (Hussnain and Afghan, 2006).

It has been reported that this disease became highly destructive in the northwestern part of the country due to favourable environmental conditions of high humidity and ideal temperature during crop season in this area (Tiwari et al., 2010). The development and use of resistant cultivars is the most reliable, long lasting, economical and integral mean for the control of this disease (Sengar et al., 2009). However, the major bottleneck in assessing durable resistance among different varieties/ genotypes to red rot is the highly variable nature of pathogen in the form of different strains, which results in frequent breakdown of host resistance under field conditions. Colletotrichum spp. infecting diverse hosts have a high degree of pathogenic variability (Sharma et al., 2005). Several epiphytotics of red rot have been reported in the past, resulted in the complete failure of many elite cultivars like Co 1148, Co 7717 and CoJ 64. The pathogen undergoes adaptive changes in relation to the host varieties cultivated, which subsequently leads to alterations in the virulence pattern of the fungus (Satyavir, 2003; Duttamajumdar, 2008).

The typical morphological and cultural features of $C$. falcatum include acervulli with setae, presence or absence of teleomorph, colony colour, sporulation and growth rate are used used for its genetic characterization (Viswanathan $e t$ al., 2003; Malathi et al., 2010). The earlier workers have designated races/ isolates of C. falcatum with emphasis on cultural and morphological characters which is time consuming and expertise specific. Thereafter, the use of differential hosts was found as a viable option for evaluation 
of pathogenic variability (Beniwal et al., 1989; Sharma et al., 1999). Since, the virulence assays are subject to environmental variations and sometimes leading to disease escape. This variability and high adaptability of the pathogen endangers the cultivation of different elite cultivars. In the recent past, various DNA- based characterization methods have been used successfully in identification of different Colletotrichum spp. infecting different hosts (Madan et al., 2000; Latha et al., 2003; Kumar et al., 2010). Therefore, present study was undertaken to apply different approaches morphological, pathological and molecular to investigate the existing variability of $C$. falcatum isolates in northern India.

\section{Materials and methods}

\section{Collection, isolation and maintenance of isolates}

Sixteen isolates of C. falcatum were isolated from red rot infected stalks of 15 sub-tropical sugarcane cultivars from different sugar mill areas of Punjab, Haryana and Uttar Pradesh (Tab. 1).

Tab. 1. Isolates of Colletotrichum falcatum used in this study and their place of collection

\begin{tabular}{cccc}
\hline S. No. & Isolate & Variety & Source of collection \\
\hline 1 & Cf-60 & CoJ-64 & Budhewal (Punjab) \\
\hline 2 & Cf-65 & Co-1148 & Dasuya (Punjab) \\
3 & Cf-157 & Co-84 & Budhewal (Punjab) \\
4 & Cf-204 & CoJ-83 & Buttar Sevian (Punjab) \\
5 & Cf-223 & CoS-88230 & Mukerian (Punjab) \\
\hline 6 & Cf-241 & Co-7717 & Fazilka (Punjab) \\
7 & Cf-245 & Co-89003 & Nawanshahr (Punjab) \\
\hline 8 & Cf-248 & CoC-90063 & Gurdaspur (Punjab) \\
\hline 9 & Cf-249 & CoJ-86 & Budhewal (Punjab) \\
\hline 10 & Cf-250 & Sel 32-00 & Gurdaspur (Punjab) \\
\hline 11 & Cf-251 & Sel 64-00 & Ladhowal (Punjab) \\
\hline 12 & Cf-252 & Sel 69-00 & Gurdaspur (Punjab) \\
\hline 13 & Cf-253 & Sel 21-00 & Ladhowal (Punjab) \\
\hline 14 & Cf-254 & CoJ-85 & Gurdaspur (Punjab) \\
\hline 15 & Cf-255 & CoS-767 & Kaithal (Haryana) \\
\hline 16 & Cf-256 & CoS-8436 & Shahjahanpur (U.P) \\
\hline
\end{tabular}

These isolates were purified by single spore isolation and maintained on oat-meal agar medium with periodic subculturing and stored at $4 \pm 1^{\circ} \mathrm{C}$ for subsequent use.

\section{Morphological and cultural variability}

The oat meal agar Petri plates were inoculated in the centre with actively growing $5 \mathrm{~mm}$ diameter mycelial disc having spores obtained from 7 days old culture and each isolate was replicated thrice. The cultures were incubated at $25 \pm 1{ }^{\circ} \mathrm{C}$ in a BOD incubator. The morphological and cultural characteristics viz., colony diameter $(\mathrm{mm})$, growth pattern, sporulation were recorded after 7 days of incubation. Sporulation intensity was calculated with the help of haemocytometer while conidial size (length and breadth) of each isolate was measured with the ocular micrometer.

\section{Pathogenicity assay}

Fourteen sugarcane differentials namely Baragua $(S$. officinarum), Khakai (S. sinense), SES 594 (S. spontaneum), CoS 767, Co 975, BO 91, CoC 671, Co 7717, Co 997, CoJ 64, Co 1148, Co 419, Co 62399 and Co 8436 were used to study pathogenic variability of $C$. falcatum under field conditions. These differentials were inoculated by plug method (Srinivasan and Bhatt, 1961) and disease data was recorded after 60 days of inoculation by splitting the canes longitudinally. Observations were recorded on 0-9 scale (Srinivasan and Bhatt, 1961) on the number of internodes transgressed by the pathogen, lesion width and white spots. The score was rated as Resistant (0-4.0), Intermediate (4.16.0) and Susceptible (> 6.1).

\section{Molecular assay}

Monoconidial cultures of all the isolates were grown on oat meal broth at $25 \pm 1{ }^{\circ} \mathrm{C}$ for 7 days. The mycelium was dried and ground to a fine powder in liquid nitrogen using pre-cooled pestle and mortar. Total genomic DNA of individual isolate was extracted using the CTAB (Cetyl trimethyl ammonium bromide) method as modified by Saghai Maroof et al (1984). Isolated DNA was purified by using $1 \mu \mathrm{L}$ RNase A $(50 \mu \mathrm{g} / \mathrm{mL})$ and resolved on $0.8 \%$ agarose gel. A working DNA solution was made by diluting the DNA stock to $25 \mathrm{ng} / \mu \mathrm{L}$ with distilled water for polymerase chain reaction (PCR) amplification.

\section{PCR amplification}

A set of 80 RAPD primers from Operon Technologies USA were first validated on single isolates. Among these 22 primers that produced consistent amplicons were further used on 16 isolates. For RAPD analysis, a $20-\mu \mathrm{L}$ PCR volume containing $2.0 \mu \mathrm{L}$ 10X buffer $(10 \mathrm{mM}$ Tris- $\mathrm{HCl}$, $\mathrm{pH} 8.0,50 \mathrm{mM} \mathrm{KCl}$ and $1.5 \mathrm{mM} \mathrm{MgCl} 2), 4.0 \mu \mathrm{L}$ dNTP mix (10 mM; Bangalore Genei), $6 \mu \mathrm{L}$ primers $(50 \mathrm{ng} / \mu \mathrm{L})$, $0.3 \mu \mathrm{L}$ Taq polymerase (Bangalore Genei; $5 \mathrm{U} / \mu \mathrm{L}$ ), $2 \mu \mathrm{L}$ DNA template $(25 \mathrm{ng} / \mu \mathrm{L})$ and $5.7 \mu \mathrm{L}$ sterilized distilled water was used. The thermal cycling profiles for RAPD were, denaturation of genomic DNA at $94^{\circ} \mathrm{C}$ for $5 \mathrm{~min}$, primer annealing at $37^{\circ} \mathrm{C}$ for $1 \mathrm{~min}$ and extension of primer at $72{ }^{\circ} \mathrm{C}$ for $2 \mathrm{~min}$. Forty additional PCR cycles were carried out at $94^{\circ} \mathrm{C}$ for $1 \mathrm{~min}, 37^{\circ} \mathrm{C}$ for 1 min and 72 ${ }^{\circ} \mathrm{C}$ for $2 \mathrm{~min}$. An additional cycle at $72^{\circ} \mathrm{C}$ for $5 \mathrm{~min}$ was run at the end of these cycles. The PCR products were resolved by electrophoresis on $1.5 \%$ agarose gel in $0.5 \mathrm{X}$ TBE buffer at $50 \mathrm{~V}$ for $3 \mathrm{~h}$. The gels were visualized under UV light and photographed using gel documentation system (UVP Transillumination).

\section{Data analysis}

The RAPD allele sizes were determined depending on the position of bands relative to the ladder $(1 \mathrm{~Kb})$. Total numbers of alleles were recorded for each RAPD marker in all the 16 isolates by giving allele number as 1, 2, 3, 4 and so on. The amplified bands were recorded as 1 (band present) or 0 (band absent) in a binary matrix. The PIC values described by Botstein et al. (1980) are used to refer the relative value of each marker with respect to the amount of polymorphism exhibited. PIC values for each of the 22 markers were estimated as per Nei (1987). Statistical analysis for RAPD data were conducted with software 
310

NTSYSpc version 2.02e (Rohlf, 1998) by using Jaccard's coefficient. Similarity was computed with SIMQUAL function for RAPD primers (binary data) and SIMINT function (interval data) for morphological/ cultural parameters using NTSYSpc version 2.02e. SHAN module of NTSYS was used for cluster analysis of both molecular (RAPD) and pathogenic data. Dendrograms were generated using Unweighted Pair Group Method based on Arithmetic average (UPGMA).

\section{Results and discussion}

\section{Morphological and cultural variability}

Visual observations showed that all the isolates used in the study possessed specific morphological features of $C$. falcatum when cultured on oat meal agar medium in Petri dishes. Based on morphological characteristics (colony colour, growth pattern, sporulation and conidial shape and size), all the 16 isolates were placed in two groups (C1- light type and $\mathrm{C} 2$ dark type). Isolates of group $\mathrm{C} 1$ had whitish grey mostly fluffy colonies produced medium or high sporulation. Twelve isolates fell in this group. Isolates of group C2 had grey, less fluffy colonies with low sporulation and 4 isolates viz., Cf-65, Cf-251, Cf-252 and Cf-256 were included in this group. Abott $(1935,1938)$ also characterized the different isolates of red rot into two distinct dark and light races on the basis of colour and texture and revealed that dark type sporulated sparingly and light type sporulated abundantly. In this study average length and width of conidia varied between 27.0-45.0 and 5.6-10.0 $\mu \mathrm{m}$, respectively. None of the isolates resembled with each other for all the morphological characters studied. However, within a single group, different isolates showed similarity in colony growth, colour and sporulation. Further, these isolates were categorized into five groups based on colony diameter. Two isolates in group I namely Cf- 157 and Cf- 249 were relatively fast growing with colony diameter ranging from $85.0-86.5 \mathrm{~mm}$ while isolate Cf-255

Tab. 2. Colony characters of different isolates of Colletotrichum falcatum in group $\mathrm{V}$ exhibited slow colony growth with diameter of $73.0 \mathrm{~mm}$ (Tab. 2). Such morphological and cultural variation in C. falcatum had been studied by earlier workers in different sugarcane varieties (Prakasam and Venkatareddy, 1961; Sharma, 1970). The findings of Jothi (1989) support the present results who divided 30 isolates of $C$. falcatum into 5 groups based on colour, texture and sporulation. The mycelial growth variation among different strains have also been documented by Viswanathan et al (2000) who worked on nine major C. falcatum pathotypes.

\section{Pathogenic variability}

Pathogenic variability of the sixteen isolates on a set of fourteen differential lines revealed that all the isolates exhibited susceptible to intermediate reaction on cvs. Khakai, CoC-671, CoJ-64 and Co-997 whereas Cvs SES594, BO-91, Co-8436 and Baragua were either resistant or intermediate to all the isolates. Six differential hosts viz., CoS-767, Co- 7717, Co-1148, Co- 419, Co- 62399 and Co-975 showed a differential behavior to red rot reaction on all the isolates (Tab. 3). Based on the disease severity (Tab. 4), isolates Cf-157, Cf-245, Cf-248 and Cf-249 were found the most virulent ( $71.43 \%$ virulence frequency) followed by Cf- 254 (64.28\%) whereas isolate Cf- 60 was the least virulent (28.58\%). Pathogenic variability in $C$. falcatum based on virulent pattern has also been reported by earlier workers who used different sets of differentials using plug and nodal methods of inoculation (Khirbat et al 1980; Beniwal et al 1989). Our results are in complete agreement with the findings of Prakasam et al (2000) who reported identification of five races of the pathogen based on differential host studies viz., Cf-1148, Cf-7717, Cf-671, Cf90063 and Cf-92061.

\section{Molecular variability}

DNA fingerprinting of all the sixteen $C$. falcatum isolates was done with 10 oligonucleotide primers. Of 80

\begin{tabular}{|c|c|c|c|c|c|c|}
\hline $\begin{array}{c}\text { Isolate } \\
\text { No. }\end{array}$ & $\begin{array}{l}\text { Colony } \\
\text { colour }\end{array}$ & $\begin{array}{c}\text { Mycelium } \\
\text { pattern }\end{array}$ & Sporulation & $\begin{array}{c}\text { Length*1 } \\
(\mu \mathrm{m})\end{array}$ & $\begin{array}{c}\text { Breadth*1 } \\
(\mu \mathrm{m})\end{array}$ & $\begin{array}{c}\text { Isolate } \\
\text { No. }\end{array}$ \\
\hline Cf-60 & ${ }^{+} \mathrm{WG}(\mathrm{L})$ & More fluffy & Medium $^{* *}$ & 45.00 & 7.5 & Cf-60 \\
\hline Cf- 65 & Grey (D) & Less fluffy & Medium & 28.75 & 7.5 & Cf-65 \\
\hline Cf-157 & ${ }^{++} \mathrm{GW}(\mathrm{L})$ & Fluffy & Low ${ }^{*}$ & 31.00 & 7.5 & Cf-157 \\
\hline Cf-204 & WG (L) & Less fluffy & Low & 27.50 & 9.0 & Cf-204 \\
\hline Cf-223 & WG (L) & Less fluffy & Low & 29.50 & 10.0 & Cf- 223 \\
\hline Cf-241 & WG (L) & Less fluffy & Medium & 27.75 & 6.5 & Cf-241 \\
\hline Cf-245 & GW (L) & Fluffy & Medium & 38.00 & 10.0 & Cf- 245 \\
\hline Cf- 248 & GW (L) & More fluffy & Medium & 29.00 & 6.5 & Cf- 248 \\
\hline Cf-249 & WG (L) & Fluffy & Medium & 29.00 & 7.5 & Cf-249 \\
\hline Cf- 250 & GW (L) & Fluffy & High $^{* * *}$ & 40.00 & 9.0 & Cf- 250 \\
\hline Cf-251 & Grey (D) & Less fluffy & Medium & 38.50 & 9.0 & Cf- 251 \\
\hline Cf- 252 & Grey (D) & Less fluffy & Medium & 34.00 & 8.5 & Cf- 252 \\
\hline Cf- 253 & WG (L) & Less fluffy & Low & 30.50 & 10.0 & Cf- 253 \\
\hline Cf- 254 & GW (L) & Fluffy & High & 27.00 & 5.6 & Cf- 254 \\
\hline Cf- 255 & WG (L) & More fluffy & Medium & 32.50 & 10.0 & Cf- 255 \\
\hline Cf-256 & Grey (D) & Fluffy & Medium & 30.00 & 6.5 & Cf- 256 \\
\hline
\end{tabular}

${ }^{*}$ Low $=2.08$ to $16.67 \times 10^{3} / 9 \mathrm{~mm}$ disc

${ }^{* *}$ Medium $=16.67$ to $29.17 \times 10^{5} / 9 \mathrm{~mm}$ disc

${ }^{* * *}$ High $=29.17$ to $41.67 \times 10^{5} / 9 \mathrm{~mm}$ disc

${ }^{+} \mathrm{WG}=$ Whitish grey; ${ }^{++} \mathrm{GW}=$ Greyish white $\mathrm{L}=$ Light; $\mathrm{D}=$ Dark

${ }^{* 1}$ Average of 50 observations 
Tab. 3. Expression of red rot isolates on sugarcane differentials by plug method of inoculation (after 60 days)

\begin{tabular}{|c|c|c|c|c|c|c|c|c|c|c|c|c|c|c|c|c|}
\hline \multirow[b]{2}{*}{$\begin{array}{l}\text { S. } \\
\text { No }\end{array}$} & \multirow[b]{2}{*}{$\begin{array}{c}\text { Isolate } \\
\text { No }\end{array}$} & \multirow[b]{2}{*}{$\begin{array}{c}\text { Host } \\
\text { variety }\end{array}$} & \multicolumn{14}{|c|}{ Differential hosts } \\
\hline & & & $\begin{array}{l}\text { Bara- } \\
\text { gua }\end{array}$ & $\begin{array}{l}\text { Kha } \\
\text { kai }\end{array}$ & $\begin{array}{c}\text { SE } \\
\text { S } \\
594 \\
\end{array}$ & $\begin{array}{l}\mathrm{CoS} \\
767\end{array}$ & $\begin{array}{c}\text { BO } \\
91\end{array}$ & $\begin{array}{c}\mathrm{CoC} \\
671\end{array}$ & $\begin{array}{c}\text { Co } \\
7717\end{array}$ & $\begin{array}{l}\text { Co } \\
997\end{array}$ & $\begin{array}{c}\mathrm{CoJ} \\
64\end{array}$ & $\begin{array}{c}\text { Co } \\
1148\end{array}$ & $\begin{array}{l}\text { Co } \\
419\end{array}$ & $\begin{array}{c}\text { Co } \\
62399\end{array}$ & $\begin{array}{l}\text { Co } \\
975\end{array}$ & $\begin{array}{c}\text { Co } \\
8436\end{array}$ \\
\hline 1 & Cf-60 & CoJ 64 & $\mathrm{R}$ & $S$ & $\mathrm{R}$ & $\mathrm{R}$ & $\mathrm{R}$ & $\mathrm{S}$ & $\mathrm{R}$ & $\mathrm{S}$ & $S$ & $\mathrm{X}$ & $\mathrm{R}$ & $\mathrm{R}$ & $\mathrm{X}$ & $\mathrm{R}$ \\
\hline 2 & Cf-65 & Co 1148 & $\mathrm{R}$ & $S$ & $\mathrm{R}$ & $\mathrm{R}$ & $\mathrm{R}$ & S & $\mathrm{R}$ & S & $S$ & $S$ & $\mathrm{X}$ & $S$ & $\mathrm{X}$ & $\mathrm{R}$ \\
\hline 3 & Cf-157 & $\mathrm{CoJ} 84$ & $\mathrm{R}$ & $S$ & $\mathrm{R}$ & $S$ & $\mathrm{R}$ & $S$ & S & $\mathrm{S}$ & $S$ & $S$ & $S$ & $\mathrm{~s}$ & s & $\mathrm{R}$ \\
\hline 4 & Cf-204 & $\mathrm{CoJ} 83$ & $\mathrm{R}$ & S & $\mathrm{R}$ & $\mathrm{R}$ & $\mathrm{R}$ & $S$ & S & S & S & $\mathrm{X}$ & S & S & S & $\mathrm{R}$ \\
\hline 5 & Cf-241 & Co 7717 & $\mathrm{R}$ & S & $\mathrm{R}$ & $\mathrm{R}$ & $\mathrm{R}$ & S & $S$ & $\mathrm{~S}$ & $\mathrm{~S}$ & $\mathrm{R}$ & $\mathrm{X}$ & $\mathrm{R}$ & $\mathrm{R}$ & $\mathrm{R}$ \\
\hline 6 & Cf-245 & $\begin{array}{c}\text { Co } \\
89003\end{array}$ & $\mathrm{R}$ & $S$ & $\mathrm{R}$ & $S$ & $\mathrm{R}$ & $S$ & $S$ & $S$ & $S$ & $S$ & $S$ & $S$ & $S$ & $\mathrm{R}$ \\
\hline 7 & Cf-223 & $\begin{array}{c}\mathrm{CoS} \\
88230\end{array}$ & $\mathrm{R}$ & $S$ & $\mathrm{R}$ & $\mathrm{R}$ & $\mathrm{R}$ & $S$ & $S$ & $S$ & $S$ & $\mathrm{X}$ & $\mathrm{X}$ & $S$ & $\mathrm{X}$ & $\mathrm{R}$ \\
\hline 8 & Cf-248 & $\begin{array}{c}\mathrm{CoC} \\
90063\end{array}$ & $\mathrm{R}$ & $S$ & $\mathrm{R}$ & $S$ & $\mathrm{R}$ & $S$ & $S$ & $S$ & $S$ & $S$ & $S$ & $S$ & $S$ & $\mathrm{R}$ \\
\hline 9 & Cf-249 & CoJ 86 & $\mathrm{R}$ & $S$ & $\mathrm{R}$ & $\mathrm{s}$ & $\mathrm{R}$ & $S$ & $S$ & $S$ & $\mathrm{~s}$ & $S$ & S & S & $\mathrm{S}$ & $\mathrm{R}$ \\
\hline 10 & Cf- 250 & $\begin{array}{c}\text { Sel 32- } \\
00\end{array}$ & $\mathrm{X}$ & $S$ & $\mathrm{R}$ & $\mathrm{R}$ & $\mathrm{R}$ & $S$ & $\mathrm{R}$ & $S$ & $S$ & $S$ & $\mathrm{R}$ & $S$ & $\mathrm{R}$ & $\mathrm{R}$ \\
\hline 11 & Cf-251 & $\begin{array}{c}\text { Sel 64- } \\
00\end{array}$ & $\mathrm{R}$ & $S$ & $\mathrm{R}$ & $\mathrm{R}$ & $\mathrm{R}$ & $S$ & $\mathrm{R}$ & $\mathrm{X}$ & $S$ & $S$ & $\mathrm{X}$ & $S$ & $\mathrm{R}$ & $\mathrm{R}$ \\
\hline 12 & Cf- 252 & $\begin{array}{c}\text { Sel 69- } \\
00\end{array}$ & $\mathrm{X}$ & $S$ & $\mathrm{R}$ & $\mathrm{R}$ & $\mathrm{R}$ & $S$ & $\mathrm{R}$ & $S$ & $S$ & $S$ & $S$ & $S$ & $\mathrm{R}$ & $\mathrm{R}$ \\
\hline 13 & Cf- 253 & $\begin{array}{c}\text { Sel 21- } \\
00\end{array}$ & $\mathrm{R}$ & $S$ & $\mathrm{R}$ & $\mathrm{R}$ & $\mathrm{R}$ & $S$ & $\mathrm{R}$ & $\mathrm{X}$ & $S$ & $S$ & $\mathrm{x}$ & $\mathrm{s}$ & $\mathrm{R}$ & $\mathrm{R}$ \\
\hline 14 & Cf- 254 & $\mathrm{CoJ} 85$ & $\mathrm{x}$ & $S$ & $\mathrm{R}$ & $\mathrm{x}$ & $\mathrm{R}$ & $S$ & $S$ & S & $S$ & $S$ & S & S & $\mathrm{X}$ & $\mathrm{R}$ \\
\hline 15 & Cf- 255 & $\operatorname{CoS} 767$ & $\mathrm{R}$ & $S$ & $\mathrm{R}$ & $S$ & $\mathrm{R}$ & $S$ & $\mathrm{R}$ & $S$ & $S$ & $S$ & $\mathrm{R}$ & $\mathrm{R}$ & $\mathrm{R}$ & $\mathrm{R}$ \\
\hline 16 & Cf-256 & $\begin{array}{c}\mathrm{CoS} \\
8436\end{array}$ & $\mathrm{x}$ & $S$ & $\mathrm{R}$ & $\mathrm{R}$ & $\mathrm{R}$ & $S$ & $\mathrm{x}$ & $S$ & $S$ & $\mathrm{X}$ & $\mathrm{R}$ & $S$ & $S$ & $\mathrm{R}$ \\
\hline
\end{tabular}

Score $0-4=$ Resistant $(\mathrm{R})$

4.1-6 = Intermediate $(\mathrm{X})$

$>6.1=$ Susceptible $(S)$

Tab. 4. Virulence frequency of isolates of Colletotrichum falcatum

\begin{tabular}{ccccc}
\hline S. No. & Isolate & Variety & Number of differentials infected & Virulence frequency (\%) \\
\hline 1 & Cf-60 & CoJ-64 & 4 & 28.58 \\
2 & Cf-65 & Co-1148 & 6 & 42.86 \\
3 & Cf-157 & Co-84 & 10 & 71.43 \\
4 & Cf-204 & CoJ-83 & 8 & 57.14 \\
5 & Cf-223 & CoS-88230 & 6 & 42.86 \\
\hline 6 & Cf-241 & Co- 7717 & 5 & 35.71 \\
7 & Cf-245 & Co-89003 & 10 & 71.43 \\
8 & Cf-248 & CoC-90063 & 10 & 71.43 \\
9 & Cf-249 & CoJ-86 & 10 & 71.43 \\
10 & Cf-250 & Sel 32-00 & 6 & 42.86 \\
11 & Cf-251 & Sel 64-00 & 5 & 35.71 \\
\hline
\end{tabular}


312

primers initially used for amplification, 22 were selected which were most polymorphic and consistently produced 10-18 amplicons (Tab. 5) of 0.3-2.7 kb size. Among these 22 primers, 10 primers were able to give unique bands in various isolates. Primer S103 amplified maximum bands (Fig. 1) while primer $S 110$ and $S 114$ amplified the least

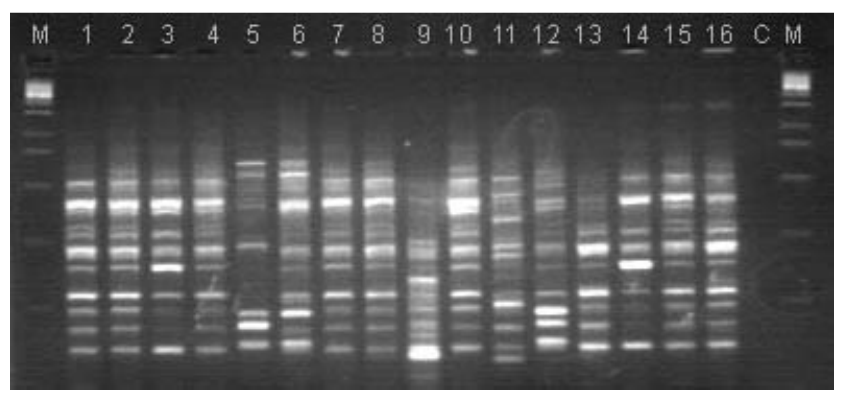

Fig. 1. PCR amplification products obtained with primer $S$ 103, Lane $M$ indicates standard DNA ladder of 100-bp marker and 1-16 indicates Cf-65, Cf-60, Cf-254, Cf-254m, Cf-223, Cf-250, Cf-249, Cf-255, Cf-248, Cf-253, Cf-256, Cf252, Cf-204, Cf-241, Cf-251 and Cf-245 respectively, C indicates control bands. Information generated from these banding patterns was used to study the genetic distance between the test isolates. Primer S119 generated specific amplification pattern in isolates Cf-204, Cf-249 and Cf-254 with single unique band while 2 unique bands were found in isolate $\mathrm{Cf}$ 248. S119 RAPD primer was more prominent and could be used to identify 13 to 16 isolates as it showed highest efficiency of $81.25 \%$. Similarity coefficient based on DNA amplification using RAPD primers was estimated using Jaccard's coefficient of similarity (Sneath and Sokal, 1973). The similarity coefficient ranged from 0.31 to 0.83 (Tab. 6). Molecular data revealed that isolate Cf-223 showed least similarity coefficient 0.31 ) with Cf-253 isolate indicating their diverse nature at molecular level while isolate Cf-204 showed high similarity (0.83) with Cf-245 indicating their close relationship. The polymorphic information content (PIC) values ranged from 0.701 (primer S1118) to 0.929 ( primer S103) with an average of 0.867 for all 22 primers. Cluster analysis was conducted to analyze the genetic similarities among different isolates as revealed by RAPD marker system (Fig. 2). At 60 per cent coefficient of similarity one major cluster was identified with eleven isolates while rest five isolates (Cf-223, Cf-248, Cf-250, Cf252 and Cf-256) showed independent lineages. This cluster was found very heterogeneous as it comprised of isolates

Tab. 5. Primers used in RAPD analysis of isolates of Colletotrichum falcatum

\begin{tabular}{|c|c|c|c|c|}
\hline S. No. & $\begin{array}{l}\text { Primer } \\
\text { Code }\end{array}$ & $\begin{array}{c}\text { Primer } \\
\text { sequence }\end{array}$ & $\begin{array}{l}\text { Polymorphic Information } \\
\text { Content (PIC) values }\end{array}$ & $\begin{array}{l}\text { No. of amplified } \\
\text { fragments }\end{array}$ \\
\hline 1 & S 103 & 5'-AGACGTCCAC-3' & 0.929 & 18 \\
\hline 2 & S 104 & 5'-GGAAGTCGCC-3' & 0.850 & 13 \\
\hline 3 & S 108 & 5'-GAAACACCCC-3' & 0.882 & 12 \\
\hline 4 & S 110 & 5'-CCTACGTCAG-3' & 0.895 & 10 \\
\hline 5 & S 111 & 5'-CTTTCCGCAGT-3' & 0.867 & 15 \\
\hline 6 & S 114 & 5'-ACCAGGTTGG-3' & 0.747 & 10 \\
\hline 7 & S 115 & 5'AATGGCGCAG-3' & 0.922 & 14 \\
\hline 8 & S 116 & 5'-TCTCAGCTGG-3' & 0.926 & 15 \\
\hline 9 & S 118 & 5'-GAATCGGCCA-3' & 0.799 & 11 \\
\hline 10 & S119 & 5'-CTGACCAGCC-3' & 0.791 & 17 \\
\hline 11 & S 1101 & 5'-TCACGTACGG-3' & 0.926 & 15 \\
\hline 12 & S 1103 & 5'-CTTCCCTGTGT-3' & 0.900 & 17 \\
\hline 13 & S 1104 & 5'-GAGGGACCTC-3' & 0.903 & 16 \\
\hline 14 & S 1105 & 5'-GGGCTATGCC-3' & 0.893 & 13 \\
\hline 15 & S 1106 & 5'-CTCGGGATGT-3' & 0.907 & 17 \\
\hline 16 & S 1108 & 5'ACCACGAGTG-3' & 0.850 & 16 \\
\hline 17 & S 1110 & 5'-CAGACCGACC-3' & 0.890 & 11 \\
\hline 18 & S 1111 & 5'AGATGCGCGG-3' & 0.927 & 16 \\
\hline 19 & S 1113 & 5'-CACGGCACAA-3' & 0.901 & 16 \\
\hline 20 & S 1114 & 5'-TGGTTGCGGA-3' & 0.788 & 14 \\
\hline 21 & S1118 & 5'ACGGGACTCT-3' & 0.701 & 17 \\
\hline 22 & $S 1120$ & 5'-ACCAACCAGG-3' & 0.877 & 13 \\
\hline
\end{tabular}

from 7 different districts of Punjab state and one from Haryana. All the isolates were associated at $40 \%$ similarity level. Isolate Cf-254 was the most diverse at molecular level as compared to other isolates. However, at 70 per cent coefficient of similarity two major clusters were identified which comprised of 11 isolates. Remaining 5 isolates showed independent lineages. Lineage I was formed by 6 isolates viz. Cf-65, Cf-60, Cf-254, Cf-254m, Cf-249 and Cf-255. Lineage II comprised of 5 isolates viz. Cf-253, Cf251, Cf-204, Cf-245 and Cf-241. One isolate Cf-256 from UP formed independent lineage $\mathrm{V}$ along with 4 isolates $\mathrm{Cf}$ 250, Cf-252, Cf-248 and Cf-223 from Punjab which formed lineage III, IV, VI and VII. Isolates from Gurdaspur formed individual lineage, indicating that $C$. falcatum isolates from Gurdaspur were genetically distinct and these are well differentiated from isolates of other regions of Punjab.

The clustering in RAPD dendrogram was not associated with the geographic locations from which the isolates were obtained. Different pathogens have been reported to possess a high degree of molecular variability when evaluated by RAPD markers like Colletotrichum accutatum (Guerber et al., 2003; Whitelaw et al., 2007), Colletotrichum gloeosporiods (Telhinhas et al., 2005). However, Thottappilly et al. (1999) 


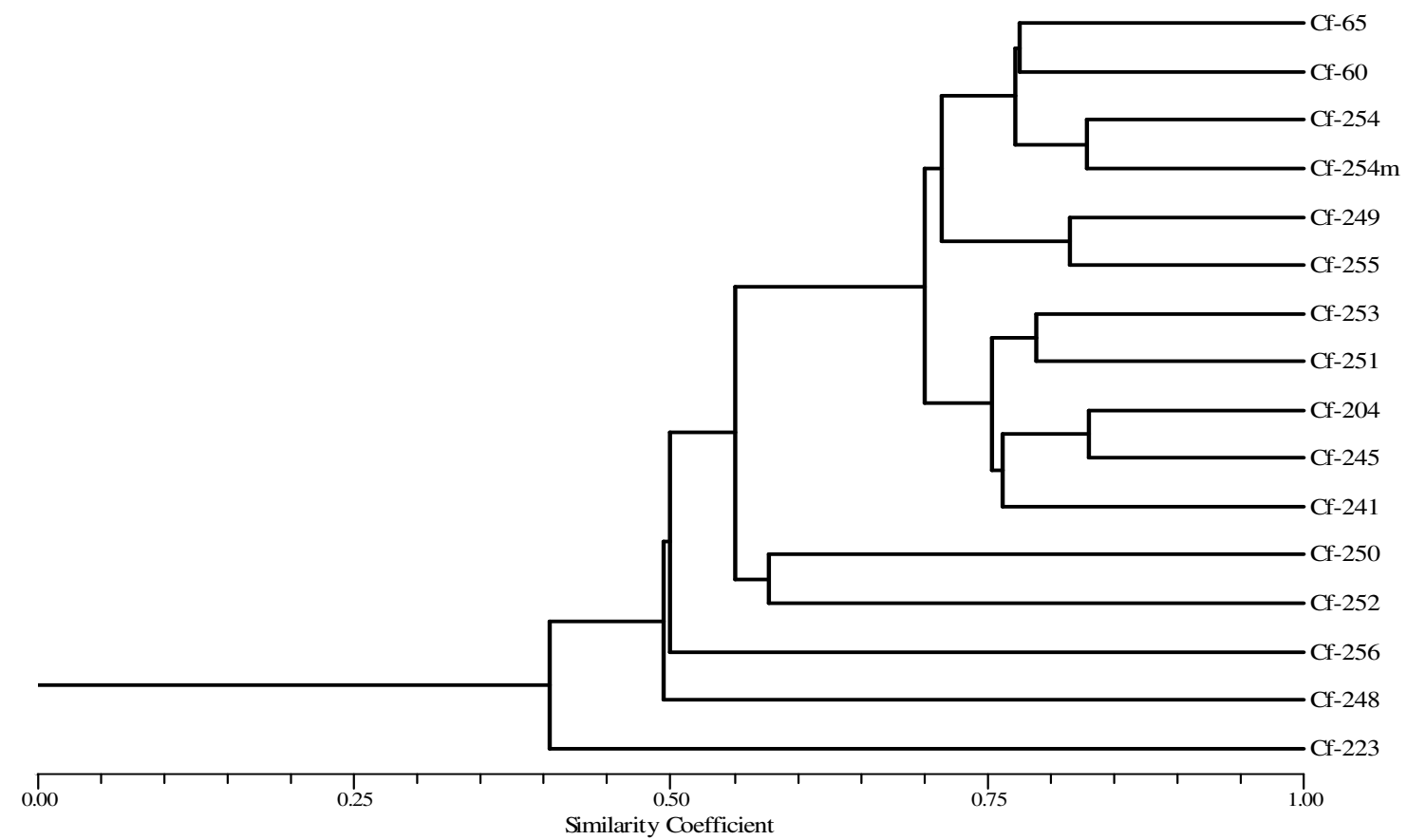

Fig. 2. Dendrogram obtained by unweighted paired group method showing molecular diversity among different isolates of Colletotrichum falcatum

Tab. 6. Similarity matrix generated by Jaccard's coefficient using molecular data

\begin{tabular}{|c|c|c|c|c|c|c|c|c|c|c|c|c|c|c|c|c|c|}
\hline No. & $\begin{array}{l}\text { Isolate } \\
\text { No. }\end{array}$ & Cf65 & $\mathrm{Cf} 60$ & Cf254 & $\mathrm{Cf} 254 \mathrm{~m}$ & Cf223 & Cf250 & Cf249 & Cf255 & Cf2 48 & Cf253 & Cf256 & Cf252 & Cf204 & Cf241 & Cf251 & $\begin{array}{c}\mathrm{Cf} 2 \\
45\end{array}$ \\
\hline 1 & Cf65 & 1.00 & & & & & & & & & & & & & & & \\
\hline 2 & Cf60 & 0.62 & 1.00 & & & & & & & & & & & & & & \\
\hline 3 & Cf254 & 0.66 & 0.66 & 1.00 & & & & & & & & & & & & & \\
\hline 4 & $\mathrm{Cf} 254 \mathrm{~m}$ & 0.67 & 0.64 & 0.74 & 1.00 & & & & & & & & & & & & \\
\hline 5 & Cf 223 & 0.00 & 0.00 & 0.00 & 0.00 & 1.00 & & & & & & & & & & & \\
\hline 6 & Cf 250 & 0.36 & 0.35 & 0.34 & 0.37 & 0.00 & 1.00 & & & & & & & & & & \\
\hline 7 & Cf 249 & 0.52 & 0.51 & 0.53 & 0.54 & 0.00 & 0.36 & 1.00 & & & & & & & & & \\
\hline 8 & Cf 255 & 0.48 & 0.52 & 0.52 & 0.54 & 0.00 & 0.37 & 0.69 & 1.00 & & & & & & & & \\
\hline 9 & Cf 248 & 0.34 & 0.30 & 0.28 & 0.32 & 0.00 & 0.27 & 0.32 & 0.33 & 1.00 & & & & & & & \\
\hline 10 & Cf 253 & 0.48 & 0.53 & 0.49 & 0.50 & 0.00 & 0.31 & 0.51 & 0.56 & 0.31 & 1.00 & & & & & & \\
\hline 11 & Cf 256 & 0.34 & 0.34 & 0.32 & 0.29 & 0.00 & 0.30 & 0.28 & 0.28 & 0.26 & 0.32 & 1.00 & & & & & \\
\hline 12 & Cf 252 & 0.33 & 0.34 & 0.30 & 0.33 & 0.00 & 0.32 & 0.30 & 0.32 & 0.22 & 0.37 & 0.27 & 1.00 & & & & \\
\hline 13. & Cf204 & 0.53 & 0.46 & 0.50 & 0.59 & 0.00 & 0.35 & 0.53 & 0.50 & 0.35 & 0.50 & 0.30 & 0.29 & 1.00 & & & \\
\hline 14. & Cf 241 & 0.57 & 0.51 & 0.55 & 0.57 & 0.00 & 0.36 & 0.55 & 0.54 & 0.30 & 0.52 & 0.31 & 0.30 & 0.60 & 1.00 & & \\
\hline 15. & Cf 251 & 0.54 & 0.48 & 0.51 & 0.54 & 0.00 & 0.36 & 0.49 & 0.52 & 0.31 & 0.65 & 0.30 & 0.33 & 0.55 & 0.62 & 1.00 & \\
\hline 16 & Cf2 245 & 0.53 & 0.54 & 0.51 & 0.55 & 0.00 & 0.34 & 0.49 & 0.54 & 0.33 & 0.59 & 0.34 & 0.32 & 0.76 & 0.63 & 0.60 & 1.00 \\
\hline
\end{tabular}

categorized 51 isolates of C. gloeosporioides into four groups using RAPD analysis, which were previously categorized on the basis of morphology and virulence. They did not find any correlation between classification of different isolates by RAPD and rate of growth of isolates in culture or their geographic origin. Padder et al. (2007) studied five $C$. lindemuthianum populations causing anthracnose in common bean to determine genetic diversity, migration and the probable rate of spread of races capable of overcoming resistance present in elite cultivars on the basis of allele frequencies of 12 RAPD markers. They suggested that naturally occurring gene flow is likely to be increased by the human activity for comprehensive cultivation of beans in the near future.

It is evident from the morphological, pathological and molecular analysis used in this study that $C$. falcatum causing red rot of sugarcane in the northern region of India possesses high degree of variability. However, it also inferred that morphological grouping of most of the isolates possess positive correlation with pathogenic variability whereas molecular diversity did not showed such correlation. The molecular analysis needs to be further validated with advanced markers like SNPs to get a précised and comprehensive results on existing variability of this pathogen for their successful exploitation in future breeding programme.

\section{Acknowledgment}

The authors would like to thank the Head of Department of Plant Breeding and Genetics and Director, School of Agricultural Biotechnology, Punjab Agricultural University, Ludhiana, for providing the facilities to carry 
314

out the present study.

\section{References}

Abbott EV (1935). Physiological specialization in Colletotrichum falcatum Went. Proc. Int Sugarcane Technol 5:730-36.

Abbott EV (1938). Red rot of sugarcane. Tech Bull U.S. Dep Agnc 641:96.

Alexander KC, Viswanathan R (1996). Major diseases affecting sugarcane production in India and recent experiences in quarantine. In: Croft BJ, Piggin CM, Wallis ES, Hogarth DM (eds.), Sugarcane Germplasm Conservation and Exchange, Canberra, Australia, Australian Centre for International Agricultural Research, Proceedings 67:46-48.

Bailey JA, Jeger MJ (1992). Colletotrichum: Biology, Pathology and Control. Wallingford, UK, CAB International.

Beniwal MS, Satyavir, Virk KS (1989). Pathogenic variability in Colletotrichum falcatum incitant of red rot of sugarcane. Indian Phytopathol 42:95-99.

Duttamajumdar SK (2008). Red Rot of Sugarcane. Lucknow India, Indian Institute of Sugarcane Research, p. 46.

Freeman S (1997). Pathogenicity and host specificity in the filamentous pathogen, Colletotrichum. Recent Advances in Microbiology. Bet Dagan, Isreal, Department of Plant Path ARO, The Volcani Center, p. 47-54.

Guerber JC, Liu B, Corell JC, Johnston PR (2003). Characterization of diversity in Colletotrichum acutatum sensu lato by sequence analysis of two gene introns, mtDNA and intron RFLPs, and mating compatibility. Mycologia 95:872-895.

Hussnain Z, Afghan S (2006). Impact of major cane diseases on sugarcane yield and sugar recovery. Jhang, Pakistan, Shakarganj Sugar Research Institute, Annual report.

Jothi R (1989). Studies on variation in red rot pathogen Colletotrichum falcatum Went. on sugarcane. Ph.D. dissertation, Bharathiyar University, Coimbatore.

Khirbat SK, Satyavir, Beniwal MS (1980). Physiological and pathological variability in sugarcane red rot pathogen, Colletotrichum falcatum in Haryana. Indian Phytopath 33:296-99.

Latha J, Chakrabarti A, Mathur K, Rao VP, Thakur RP, Mukherjee PK (2003). Genetic diversity of Colletotrichum graminicola isolates from India revealed by restriction analysis of PCR-amplified intergenic spacer region of nuclear rDNA. Curr Sci 84:881-883.

Madan VK, Bikas M, Ansari ML, Srivastava A, Soni N, Solaman S, Agnihotri VP (2000). RAPD-PCR analysis of molecular variability in the red rot pathogen (Colletotrichum falcatum) of sugarcane. Sugarcane Int 3:58.

Malathi P, Viswanathan R, Ramesh Sundar A, Prakasam N, Padmanaban P, Jothi R, Renuka Devi SR, Poongothai M (2010). Variability among Colletotrichum falcatum pathotypes used for screening red rot resistance in sugarcane. Sugarcane Int 28(2):47-52.

Nei M (1987). Molecular Evolutionary Genetics. Columbia University Press, New York. p. 645.

Padder BA, Sharma PN, Sharma OP, Kapoor V (2007). Genetic diversity and gene flow estimates among five populations of Colletotrichum lindemutbianum across Himachal Pradesh. Physiol Mol Plant Pathol 70:8-12.

Prakasam N, Padmanaban P, Mohanraj D (2000). Identification of red rot pathotypes using differential host reaction. Ann Report for 1999-2000. Sugarcane Breeding Institute, Coimbatore, p. 44.

Prakasam R, Venkatareddy TC (1961). Occurrence of light race of Colletotrichum falcatum Went in Andhra Pradesh. Science and Culture 27:250-51.

Rafay SA, Singh VB (1957). A new strain of Glomerella tucumanesis. Curr Sci 22:19-20.

Rohlf FJ (1998). NTSYSpC numerical taxonomy and multivariate analysis, version 2.0. Applied Biostatistics Inc., New York.

Saghai-Maroof MA, Soliman KM, Jorgensen AR, Allard RW (1984). Ribosomal DNA spacers length polymorphism in barley: Mendelian inheritance, chromosomal location and population dynamics. Proc Natl Acad Sci USA 81:801418.

Saiki RK, Gelfand DH, Stoffel S, Scharf SJ, Higushi R, Hom GT, Mullis KS, Erlich HA (1988). Primer directed enzymatic amplification of DNA with a thermostable DNA polymerase. Science 239:487-91.

Satyavir (2003). Red rot of sugarcane - Current scenario. Indian Phytopathol 56:245-254.

Satyavir, Singh N, Virk KS, Nageshwar, Rao GV, Singh H, Mishra SR (2001). Pathogenic variability in sugarcane red root system. In: Nagarajan S, Singh OP (eds.), Proc Nat Symp. Role of Resistance in Intensive Agriculture, Kalyani Publishers, Ludhiana, p 109-114.

Sengar AS, Thind KS, Kumar B, Pallavi M, Gosal SS (2009). In vitro selection at cellular level for red rot resistance in sugarcane (Saccharum sp.). Plant Growth Regul 58:201209.

Sharma M N (1970). Occurrence of new strain of Physalospora tucumanesis Speg. in Nizamabad District of Andhra Pradesh. Science and Culture 36:52-54.

Sharma PN, Kaur M, Sharma OP, Sharma P, Pathania A (2005). Morphological, pathological and molecular variability of Colletotrichum capsici, the cause of fruit rot of chilies in the subtropical region of north western India. J Phytopathol 153:232-237.

Sneath PHA, Sokal RR (1973). Numerical Taxonomy. Freeman W and Co., San Francisco.

Srinivasan KV, Bhatt NR (1961). Red rot of sugarcane criteria for grading resistance. J Indian Bot Soc 11:566-77.

Suman A, Lal S, Shasany AK, Gaur A, Singh P (2005). Molecular assessment of diversity among pathotypes of 
Colletotrichum falcatum prevalent in sub-tropical Indian Viswanathan R, Samiyappan R (2000). Red rot disease in sugarcane. World J Microbiol Biotech 21:1135-1140. sugarcane: challenges and prospects. Madras Agric J 87:549-59.

Telhinhas P, Sreenivasaprasad S, Martins NJ, Oliveira H (2005). Molecular and phenotypic analysis reveals association of diverse Colletotrichum acutatum groups and low level of C. gloeosporioides with olive anthracnose. Appl Environ Microbiol 71:2987-2998.

Thottappily G, Mignouna HD, Onasanya A, Abang M, Oyelakin O, Singh NK (1999). Identification and differentiation of isolates of Colletotrichum gloeosporioides from yam by random amplified polymorphic DNA markers. African Crop Sci J 7:195-205.

Tiwari AK, Bharti YP, Mishra N, Tripathi S, Lal M, Sharma PK, Rao GP, Sharma ML (2010). Biotechnological approaches for improving sugarcane crop with special reference to disease resistance. Acta Phytopathol Entomol Hung 45(2):235-249.

Viswanathan R, Malathi P, Padmanaban P (2003). Variation in sugarcane red rot pathogen Colletotrichum falcatum Went. In: Rao GP, Manoharachari C, Bhat DJ, Rajak RC, Lakhanpal TN (eds.), Frontiers of Fungal Diversity in India, p. 639-67.

Whitelaw MAW, Curtin SJ, Huang R, Steel CC, Blanchard CL, Roffey PE (2007). Phylogenetic relationships and pathogenicity of Colletotrichum acutatum isolates from grape in subtropical Australia. Plant Pathol 56:448-463. 